\title{
Collective transport through charge-density-wave heterostructures
}

\author{
B. Rejaei and G. E. W. Bauer \\ Theoretical Physics Group, Department of Applied Physics/DIMES, Delft University of Technology, \\ Lorentzweg 1, 2628CJ Delft, The Netherlands
}

(Received 10 April 1996)

\begin{abstract}
We calculate the electric conductance of a normal-metal/charge-density-wave/normal-metal heterostructure in the sliding regime. The collective transport is understood in analogy with Andreev scattering at normalmetal/superconductor interfaces, and is expressed in terms of the local density of states and the quasiparticle transmission probability. At low temperatures the resistance due to disorder originates from the immediate vicinity of the contacts. [S0163-1829(96)02532-5]
\end{abstract}

The conductance mechanism of quasi-one-dimensional conductors exhibiting charge-density-wave (CDW) transitions has been the subject of many theoretical and experimental studies. ${ }^{1}$ In particular, the discovery of narrow-band noise $^{2,3}$ and nonlinear current-voltage characteristics in these systems has attracted much attention. In order to explain these phenomena, a number of theories were proposed which can be roughly categorized as phenomenological models, ${ }^{4}$ semimicroscopic treatments based on the phase Hamiltonian, ${ }^{5-7}$ and microscopic calculations. ${ }^{8,9}$ From a formal point of view there are many similarities between these theories and the theory of superconductivity. However, despite the extensive literature on mesoscopic superconducting heterostructures, almost no similar studies exist for CDW systems. Recently, the group Mooij at Delft started a project of film growth and structuring for the fabrication of thin, mesoscopic CDW films. Motivated by these advances, Visscher and Bauer ${ }^{10}$ developed a mean-field theory for the ground state of CDW heterostructures and Tanaka et al. ${ }^{11}$ studied the quasiparticle transport in normal-metal/insulator/ CDW junctions.

In this paper we investigate the conductance properties of a normal-metal/CDW/normal-metal $(N / C / N)$ heterostructure with emphasis on the collective motion of the CDW condensate. Our model consists of parallel one-dimensional CDW chains of length $L$ perpendicular to the interfaces with two normal, low impedance leads (cf. Fig. 1), which are connected to two large reservoirs at the chemical potentials $\mu_{L}$ and $\mu_{R}$. We restrict ourselves to CDW compounds with a semiconducting spectrum below the transition temperature $\mathcal{T}_{P}$ (examples are $\mathrm{TaS}_{3}$ and $\mathrm{K}_{0.3} \mathrm{MoO}_{3}$ ), and assume the coupling between the chains to be small. ${ }^{12}$ The CDW then consists of a lattice distortion coupled to an electron density modulation $n_{\mathrm{CDW}} \propto|\Delta(x, t)| \cos \left[2 k_{F} x-\chi(x, t)\right]$ where $k_{F}$ is the Fermi wave number and $|\Delta|$ and $\chi$ are the time- and spacedependent modulus and phase of the CDW order parameter, respectively. $|\Delta|$ is one-half of the Peierls energy gap at the Fermi energy.

We shall restrict ourselves to the incommensurate case where the free motion of the condensate is only prohibited by impurities. ${ }^{13}$ When the potential difference $\delta \mu=\mu_{L}-\mu_{R}$ is below the threshold voltage $\delta \mu_{T}$, the CDW is pinned and the current is carried by the quasiparticles above the energy gap (comparable to the normal current in superconductors). Above the threshold voltage the CDW slides along the sample with velocity $v_{\mathrm{CDW}}=\dot{\chi} / 2 k_{F}$ $\left(\dot{\chi} \equiv \partial_{t} \chi\right)$. The sliding motion of the CDW, whether local or global, is seen as the main source of the narrow-band noise. ${ }^{1}$

Using the method of kinetic equations ${ }^{14}$ based on the Keldysh formalism, ${ }^{15}$ we derive expressions for the conductance of the $N / C / N$ junction in the so-called sliding regime ${ }^{9}$ where $\delta \mu \gg \delta \mu_{T}$. It is shown that at zero temperature the sliding CDW does not experience any friction from the bulk density of impurities, i.e., resistance is only caused by the disorder near the contacts. This result is then interpreted by using the analogy with Andreev reflection in superconductivity. We also consider the CDW slightly below $\mathcal{T}_{P}$ and calculate the correction to the conductance of the normal metal as a result of CDW formation in the dirty and clean limits.

We first present a brief discussion of the method of kinetic equations ${ }^{9,14}$ which provides a consistent theoretical framework for the semiclassical dynamics of the system. The motion of the quasiparticles and the condensate in a CDW system can be described by the Green functions $g_{\alpha \beta}^{i}\left(x ; t, t^{\prime}\right)$ where $i=\{R, A, K\}$ and $\alpha, \beta=\{1,2\}$. The retarded $g^{R}$ and advanced $g^{A}$ functions determine the excitation spectrum, and the Keldysh function $g^{K}$ describes the

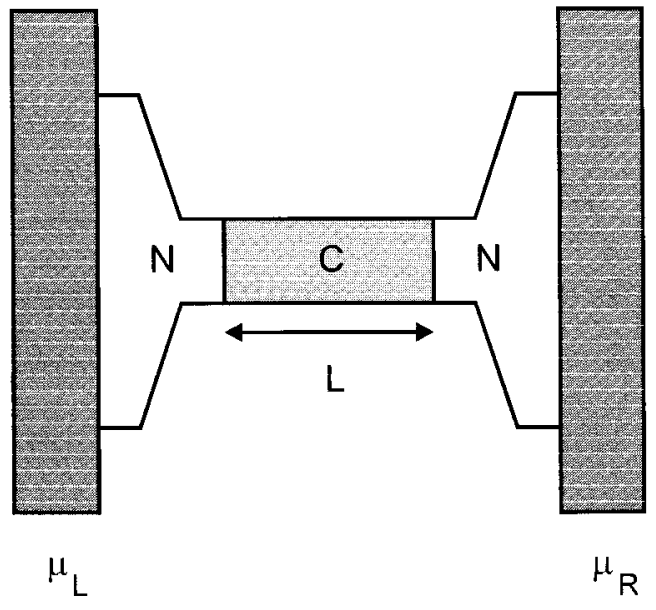

FIG. 1. The $N / C / N$ heterostructure. A thin CDW film of length $L$ is connected to two perfectly conducting normal leads. The leads are connected to two reservoirs at chemical potentials $\mu_{L}$ and $\mu_{R}$. 
kinetics of the system. The subscripts 1 and 2 refer to the right-, respectively, left-moving electrons at the two branches of the linearized kinetic-energy spectrum. The Green functions satisfy the equation of motion

$$
i \hbar v_{F} \partial_{x} \mathbf{g}+\mathbf{H} \bigcirc \mathbf{g}-\mathbf{g} \bigcirc \mathbf{H}=0,
$$

and the normalization condition

$$
\mathbf{g} \bigcirc \mathbf{g}=\mathbf{1} \delta\left(t-t^{\prime}\right),
$$

where

$$
\begin{gathered}
\mathbf{H}=\left(i \hbar \partial_{t}-\Phi\right) \boldsymbol{\sigma}_{3}+\boldsymbol{\Delta}-\boldsymbol{\Sigma}, \\
\mathbf{g}=\left(\begin{array}{cc}
g^{R} & g^{K} \\
0 & g^{A}
\end{array}\right), \quad \boldsymbol{\Sigma}=\left(\begin{array}{cc}
\Sigma^{R} & \Sigma^{K} \\
0 & \Sigma^{A}
\end{array}\right), \\
\boldsymbol{\Delta}=\left(\begin{array}{ll}
\hat{\Delta} & 0 \\
0 & \hat{\Delta}
\end{array}\right), \quad \boldsymbol{\sigma}_{k}=\left(\begin{array}{cc}
\sigma_{k} & 0 \\
0 & \sigma_{k}
\end{array}\right) .
\end{gathered}
$$

Here $v_{F}$ is the Fermi velocity, $\Phi$ is the quasiparticle potential including the self-consistent Hartree term, $\Sigma^{i}$ is the impurity part of the self-energy, $\sigma_{k}(k=\{1,2,3\})$ are the Pauli matrices, and the operator $\bigcirc$ implies internal time integration as well as matrix multiplication. The matrix $\hat{\Delta}$ is given by $\hat{\Delta}_{11}=\hat{\Delta}_{22}=0, \hat{\Delta}_{12}=\Delta, \hat{\Delta}_{21}=-\Delta^{*}$, where the order parameter $\Delta=|\Delta| \exp (i \chi)$ is determined from the selfconsistency relation

$$
\left(1-\omega_{0}^{-2} \partial_{t}^{2}\right) \Delta(x, t)=\lambda g_{12}^{K}(x ; t, t) .
$$

Here $\lambda=\pi N(0) g_{0}^{2} / 4 \omega_{0}^{2}$ where $N(0)$ is the density of states at the Fermi energy, $\omega_{0}$ is the phonon frequency at $2 k_{F}$, and $g_{0}$ is the electron-phonon coupling constant.

The impurity scattering is treated within the selfconsistent Born approximation where

$$
\boldsymbol{\Sigma}=-\frac{1}{2} i\left(\nu_{1} \boldsymbol{\sigma}_{3} \mathbf{g} \boldsymbol{\sigma}_{3}-\frac{1}{2} \nu_{2} \boldsymbol{\sigma}_{1} \mathbf{g} \boldsymbol{\sigma}_{1}-\frac{1}{2} \nu_{2} \boldsymbol{\sigma}_{2} \mathbf{g} \boldsymbol{\sigma}_{2}\right),
$$

with $\nu_{1}$ and $\nu_{2}$ the forward and backward scattering rates, respectively. This choice of $\boldsymbol{\Sigma}$ is inevitable within the framework of kinetic equations, but implies the neglect of pinning of the condensate. ${ }^{9}$ Hence the calculation presented below is only valid in the sliding regime where the applied bias is sufficiently larger than the threshold value.

It is convenient to gauge away the phase $\chi$ in Eq. (1) by applying the unitary transformation

$$
\{\mathbf{g}, \mathbf{\Sigma}\} \rightarrow\{\widetilde{\mathbf{g}}, \widetilde{\Sigma}\}=\mathbf{U}^{\dagger}(x, t)\{\mathbf{g}, \mathbf{\Sigma}\} \mathbf{U}\left(x, t^{\prime}\right),
$$

where $\mathbf{U}=\exp \left(\frac{1}{2} \boldsymbol{i} \boldsymbol{\sigma}_{3} \chi\right)$. This is equivalent to a transformation from the reference frame of the laboratory to that of an observer moving with the velocity $v_{\mathrm{CDW}}=\dot{\chi} / 2 k_{F}$. Below we disregard the local variations of $\dot{\chi}$ and look for a stationarystate solution of the form $\widetilde{\mathbf{g}}\left(x ; t-t^{\prime}\right)$ which can be treated by the Fourier transformation

$$
\widetilde{\mathbf{g}}(x, \varepsilon)=\int d t \exp (i \varepsilon t / \hbar) \widetilde{\mathbf{g}}(x ; t) .
$$

Substitution of Eqs. (6) and (7) into Eq. (1) yields the stationary-state equation of motion

$$
i \hbar v_{F} \partial_{x} \widetilde{\mathbf{g}}+\left[(\varepsilon-\widetilde{\Phi}) \boldsymbol{\sigma}_{3}-\mathrm{i}|\Delta| \boldsymbol{\sigma}_{2}-\widetilde{\Sigma}, \widetilde{\mathbf{g}}\right]=0,
$$

where $\widetilde{\Phi}=\Phi+\frac{1}{2} \hbar v_{F} \partial_{x} \chi$. Furthermore, the normalization condition (2) is now reduced to the ordinary matrix equation $[\widetilde{\mathbf{g}}(x, \varepsilon)]^{2}=\mathbf{1}$. So far the treatment parallels that for the bulk CDW.

Equation (8) has to be supplemented by boundary conditions at $x=0$ and $x=L$ describing the normal-metal/CDW interface. In this paper we restrict ourselves to the ideal case where no defects or potential barriers are present at the contacts. The boundary conditions for the retarded $\widetilde{g}^{R}$ and advanced $\widetilde{g}^{A}$ components are then given by

$$
\begin{gathered}
\left.\widetilde{g}_{11}^{R}\right|_{x=0, L}=-\left.\widetilde{g}_{22}^{R}\right|_{x=0, L}=1, \\
\left.\widetilde{g}_{11}^{A}\right|_{x=0, L}=-\left.\widetilde{g}_{22}^{\mathrm{A}}\right|_{x=0, L}=-1, \\
\left.\widetilde{g}_{12}^{R}\right|_{x=0}=\left.\widetilde{g}_{21}^{R}\right|_{x=L}=\left.\widetilde{g}_{21}^{A}\right|_{x=0}=\left.\widetilde{g}_{12}^{A}\right|_{x=L}=0 .
\end{gathered}
$$

Equations (9a) and (9b) follow from the continuity of the Green functions at the normal-metal/CDW interfaces and the results ${ }^{16}$ for a normal metal. Equation $(9 \mathrm{c})$ is a consequence of the absence of backscattering in the leads for electrons moving away from the CDW. The corresponding nondiagonal elements of $\widetilde{g}^{R}$ and $\widetilde{g}^{A}$ (describing backscattering) will then vanish, leading to Eq. $(9 \mathrm{c})$.

The boundary conditions for $\widetilde{g}^{K}$ are determined as follows. The right- (left-) moving electrons in the left (right) lead are injected by the reservoir at the potential $\mu_{L}\left(\mu_{R}\right)$. The local distribution of right- and left-moving electrons among single-particle states is determined by the diagonal elements $g_{11}^{K}$ respectively, $g_{22}^{K}$ After taking account of the gauge transform (6) and the Fourier transform (7) we end up with the boundary conditions

$$
\begin{aligned}
& \tilde{g}_{11}^{K}(0, \varepsilon)=2-4 f\left(\varepsilon-\mu_{L}-\frac{1}{2} \hbar \dot{\chi}\right), \\
& \tilde{g}_{22}^{K}(L, \varepsilon)=2-4 f\left(\varepsilon-\mu_{R}+\frac{1}{2} \hbar \dot{\chi}\right),
\end{aligned}
$$

where $f(\varepsilon)$ is the Fermi-Dirac distribution function. Equation (10) simply states that for an observer moving with the velocity $v_{\mathrm{CDW}}$ the kinetic energy of right- and left-moving electrons is modified by $-\frac{1}{2} \hbar \dot{\chi}$ and $\frac{1}{2} \hbar \dot{\chi}$, respectively.

In principle Eqs. (8)-(10) and the self-consistency relation (4) can be used to compute the conductance $G=-e I / \delta \mu$ where $-e$ is the electron charge and

$$
I=\frac{1}{8} e v_{F} N(0) \int d \varepsilon \operatorname{tr} \widetilde{g}^{K}
$$

is the electric current. In practice, however, the system of Eqs. (8)-(10) cannot be solved analytically. Therefore we apply perturbation theory by taking $\delta \mu$ and $\dot{\chi}$ to be small, and linearize Eq. (8) around the equilibrium solution in which $\delta \mu=\dot{\chi}=0 .{ }^{17}$ The details of the calculation are given elsewhere. ${ }^{18}$ Here we present the final result for the (linear) conductance:

$$
\begin{gathered}
G=G_{\mathrm{CDW}}+G_{N}, \\
\frac{G_{\mathrm{CDW}}}{G_{0}}=\frac{\left[1+\int d \varepsilon r(\varepsilon) T(\varepsilon) \partial_{\varepsilon} f\right]^{2}}{1-\int d \varepsilon\left[D(\varepsilon)-r^{2}(\varepsilon) T(\varepsilon)\right] \partial_{\varepsilon} f},
\end{gathered}
$$




$$
\frac{G_{N}}{G_{0}}=-\int d \varepsilon T(\varepsilon) \partial_{\varepsilon} f,
$$

where $G_{0}=\frac{1}{2} e^{2} v_{F} N(0)$ is the conductance of a ballistic, normal system, $T(\varepsilon)$ is the probability for an electron at the energy $\varepsilon$ to tunnel through the CDW region, and

$$
\begin{gathered}
r(\varepsilon)=1+\frac{1}{\ell N(0)} \int_{0}^{L} d x \rho(x, \varepsilon), \\
D(\varepsilon)=\frac{1}{\ell[N(0)]^{2}} \int_{0}^{L} d x[\rho(x, \varepsilon)]^{2},
\end{gathered}
$$

where $\ell=\hbar v_{F} / \nu_{2}$ is the elastic mean free path and $\rho(x, \varepsilon)$ is the local equilibrium density of states. In Eq. (12) $G_{\mathrm{CDW}}$ and $G_{\mathrm{N}}$ denote the contributions of the condensate and the quasiparticles, respectively. Equations (12) and (13) can be used to study the behavior of the $N / C / N$ system in two important limiting cases: the zero temperature limit $\mathcal{T}=0$ where $\nu_{1,2} \ll|\Delta|$ and near the CDW transition temperature $\mathcal{T}_{P}$ where $\nu_{1,2} \gg|\Delta|$.

We first consider the $N / C / N$ system at $\mathcal{T}=0$, thus restricting ourselves to quantities at the Fermi energy by $\partial_{\varepsilon} f=-\delta(\varepsilon)$ in Eq. (12). At $\mathcal{T}=0$ the local density of states $\rho(x, 0)$ decays exponentially inside the CDW region, where a large energy gap $(\sim 2|\Delta|)$ opens. Consequently, the transmission probability $T(0)$ becomes exponentially small and the current is carried by the condensate only. Hence from Eq. (12) we have

$$
\frac{G}{G_{0}} \sim \frac{G_{\mathrm{CDW}}}{G_{0}} \sim \frac{1}{1+D(0)} .
$$

From the definition of $D$ [cf. Eq. (13)] and Eq. (14) we conclude that at $\mathcal{T}=0$ the conductance of the $N / C / N$ system is not affected by the bulk concentration of impurities. The condensate, driven by $\delta \mu$, experiences friction only near the contacts where the density of states at the Fermi energy does not vanish immediately.

In order to determine $D(0)$, we have to calculate the density of states at the Fermi energy $\rho(x, 0)$. This calculation is complicated by the spatial variations of $\Delta$ near the contacts (proximity effect). For simplicity we use here the stepfunction model

$$
\Delta(x)=\left\{\begin{array}{cc}
\Delta, & 0<x<L \\
0, & x<0, \quad x>L
\end{array}\right.
$$

which agrees quite well with self-consistent calculations. ${ }^{10}$ To first order in $\nu_{2} /|\Delta|=\xi / \ell\left(\xi=\hbar v_{F} /|\Delta|\right.$ is the coherence length of the CDW) (Ref. 18)

$$
\frac{\rho(x, 0)}{N(0)}=\frac{\cosh (z)}{\cosh (\zeta)}\left[1-\left(\frac{\xi}{\ell}\right)\left(\frac{\nu_{0}}{4 \nu_{2}}\right)[z \tanh (z)-\zeta]\right],
$$

where $z=(2 x-L) / \xi, \zeta=L / \xi, \nu_{0}=2 \nu_{1}+\nu_{2}$. After substituting Eq. (16) into Eqs. (13) and (14) we find

$$
\frac{G}{G_{0}} \sim 1-\frac{1}{2}\left(\frac{\xi}{\ell}\right)-\left[\frac{1}{4}+\frac{\nu_{0}}{8 \nu_{2}}\right]\left(\frac{\xi}{\ell}\right)^{2}+\cdots .
$$

As indicated by Eq. (17), dissipation is restricted to narrow regions (with lengths of the order of $\xi$ ) near the contacts, reducing the conductance of an otherwise ballistic system only weakly.

This result can be interpreted by using the analogy with Andreev reflection in superconductivity. The CDW at low temperatures behaves as a rigid condensate of electron-hole pairs. Due to the energy gap, an electron near the Fermi surface cannot propagate through the CDW region unless it simultaneously drags a hole from the Fermi sea, forming a new electron-hole pair. The latter then "freezes" into the condensate as the CDW moves away from the interface, whereas an extra electron is reflected back into the Fermi sea. The momentum of the reflected electron is slightly less than that of the original electron: the difference is transferred to the moving condensate. Hence, although charge is conserved, momentum is not. ${ }^{19}$ At the other interface the opposite process takes place. As the CDW approaches the interface, the condensate "melts" by the destruction of electronhole pairs: holes recombine with the electrons in the Fermi sea while electrons propagate freely in the normal region. Because of the energy gap, the disorder can only generate friction near the contacts where solidification and melting take place.

We next consider the limit $\mathcal{T}_{P}-\mathcal{T} \ll \mathcal{T}_{P}$ where $\nu_{1,2} \gg|\Delta|$. Although $\Delta$ is not zero in this case, the energy gap is destroyed by the impurities, leading to finite bulk density of states and transmission probability at the Fermi energy. (This situation is analogous to that of gapless superconductivity, where magnetic impurities destroy the energy gap at sufficiently high temperatures.) Due to the absence of an energy gap, the dissipation is now mainly a bulk phenomenon, in contrast to the low temperature case. Hence we may disregard the $x$ dependence of $\rho$ and use the results for a uniform system. To second order in $\ell / \xi=|\Delta| / \nu_{2}$ we have ${ }^{18}$

$$
\begin{gathered}
\frac{\rho(\varepsilon)}{N(0)}=1+\left(\frac{\ell}{\xi}\right)^{2} \frac{2 \nu_{2}^{2}\left(4 \varepsilon^{2}-\nu_{0}^{2}\right)}{\left(4 \varepsilon^{2}+\nu_{0}^{2}\right)^{2}}, \\
T(\varepsilon)=\frac{1}{R}-\left(\frac{\ell}{\xi}\right)^{2}\left(\frac{R-1}{R^{2}}\right) \frac{2 \nu_{0} \nu_{2}}{4 \varepsilon^{2}+\nu_{0}^{2}},
\end{gathered}
$$

where $R=1+L / \ell$. Substituting Eq. (18) in Eqs. (12) and (13) and considering the clean $\left(\nu_{1,2} \ll k_{B} \mathcal{T}_{P}\right)$ and dirty $\left(\nu_{1,2} \gg k_{B} \mathcal{T}_{P}\right)$ limits we obtain $G_{N} / G_{0}=1 / R$ and

$$
\begin{gathered}
\frac{G_{\mathrm{CDW}}}{G_{0}} \sim \frac{\pi}{4 \sqrt{2}}\left(\frac{R-1}{R^{2}}\right)\left(\frac{|\Delta|}{k_{B} \mathcal{T}_{P}}\right)^{2}, \quad \nu_{1,2} \ll k_{B} \mathcal{T}_{P} \\
\frac{G_{\mathrm{CDW}}}{G_{0}} \sim 2\left(1+\frac{\nu_{2}}{\nu_{0}}\right)\left(\frac{R-1}{R^{2}}\right)\left(\frac{|\Delta|}{\nu_{0}}\right)^{2}, \quad \nu_{1,2} \gg k_{B} \mathcal{T}_{P} .
\end{gathered}
$$

Since in the gapless regime $|\Delta| \propto\left(\mathcal{T}_{P}-\mathcal{T}\right)^{1 / 2}$ (see Gor'kov in Ref. 1), $G_{\mathrm{CDW}} \propto\left(\mathcal{T}_{P}-\mathcal{T}\right)$ in both clean and dirty limits. Because of the absence of the energy gap, the enhancement $G_{\mathrm{CDW}}$ of the normal conductance should be attributed to the reduction of the bulk density of states near the Fermi energy, as seen from Eq. (18a).

In deriving the above results we (i) disregarded the pinning effects, (ii) disregarded the local variations of the slid- 
ing velocity $\dot{\chi}$, and (iii) assumed perfectly conducting leads and ideal contacts. As regards point (i) it should be noted that CDW pinning requires a treatment beyond the selfconsistent Born approximation (5). An alternative approach, currently pursued by the authors, is to incorporate a few dominating pinning sites by extra boundary conditions on the Green functions.

The second simplification (neglect of the spatial variations of $\dot{\chi}$ ) is valid when the condensate is moving as a whole, but fails to explain intricate phenomena such as the motion of phase-slip centers ${ }^{1}$ which involves a nonuniform distribution of $\dot{\chi}$.

Finally, we assumed perfect leads and disregarded all scattering sources at the interface. This is certainly not true in experiments where high-electron-density contacts are used. Differences in electron density between the CDW and the contacts correspond to large potential steps at the interfaces. This problem can be settled by considering a geometry in which the CDW is separated from the contacts by two disordered normal regions with the same electron density as that of the CDW (e.g., the CDW material in the normal state). The coherent Friedel oscillations in these regions are then destroyed by the disorder ${ }^{10}$ so that despite the potential steps at the contacts, the conductance properties of the CDW are not affected. The results presented above should then remain valid provided that $T(\varepsilon)$ in Eq. (12) describes the transmission probability through the whole configuration.

We conclude by summarizing the results. Using the method of kinetic equations we expressed the conductance of the $N / C / N$ system in the sliding regime in terms of the local density of states $\rho(x, \varepsilon)$ and the transmission probability $T(\varepsilon)$. At low temperatures the $N / C / N$ junction almost behaves like a normal, ballistic system: the resistance due to disorder originates only from the immediate vicinity of the contacts (or the leads). This result can be interpreted by using the analogy with Andreev reflection in superconductivity. We also considered the $N / C / N$ system slightly below the transition temperature and calculated the enhancement of the conductance due to the CDW formation in the dirty and clean limits.

This work is part of the research program of the "Stichting voor Fundamentele Onderzoek der Materie (FOM)" which is financially supported by the "Nederlandse Organisatie voor Wetenschappelijk Onderzoek (NWO)." The authors wish to thank Yu. V. Nazarov, M. I. Visscher, C. Dekker, and J. E. Mooij for many valuable discussions.

${ }^{1}$ For a review see Charge Density Waves in Solids, edited by L. P. Gor'kov and G. Grüner (North-Holland, Amsterdam, 1989). See also Electronic Properties of Quasi-One-Dimensional Materials, edited by P. Monceau (Reidel, Dordrecht, 1985), Part II, p. 139.

${ }^{2}$ R. M. Fleming and C. G. Grimes, Phys. Rev. Lett. 42, 1423 (1979).

${ }^{3}$ P. Monceau, J. Richard, and M. Renard, Phys. Rev. Lett. 45, 43 (1980); Phys. Rev. B 25, 931 (1982).

${ }^{4}$ G. Grüner, A. Zawadowski, and P. M. Chaikin, Phys. Rev. Lett. 46, 511 (1981).

${ }^{5}$ H. Fukuyama and P. A. Lee, Phys. Rev. B 17, 535 (1977).

${ }^{6}$ P. A. Lee and T. M. Rice, Phys. Rev. B 19, 3970 (1979).

${ }^{7}$ L. Sneddon, M. C. Cross, and D. S. Fisher, Phys. Rev. Lett. 49, 292 (1982).

${ }^{8}$ T. M. Rice, P. A. Lee, and M. C. Cross, Phys. Rev. B 20, 1345 (1979).

${ }^{9}$ S. N. Artemenko and A. F. Volkov, Zh. Éksp. Teor. Fiz. 80, 2018 (1981) [Sov. Phys. JETP 53, 1050 (1980)].

${ }^{10}$ M. I. Visscher and G. E. W. Bauer, Phys. Rev. B (to be published).

${ }^{11}$ Y. Tanaka, M. I. Visscher, B. Rejaei, and G. E. W. Bauer (unpublished).

${ }^{12}$ This means that $v_{\perp} \ll v_{F}$ where $v_{\perp}$ is the transverse velocity of electrons. The coupling, however, should not be too small since otherwise thermal fluctuations and localization effects will dominate.

${ }^{13}$ P. A. Lee, T. M. Rice, and P. W. Anderson, Solid State Commun. 14, 703 (1974).

${ }^{14}$ A. I. Larkin and Yu. N. Ovchinnikov, Zh. Éksp. Teor. Fiz. 73, 299 (1977) [Sov. Phys. JETP 46, 155 (1977)].

${ }^{15}$ L. V. Keldysh, Zh. Éksp. Teor. Fiz. 47, 1515 (1964) [Sov. Phys. JETP 20, 1018 (1964)].

${ }^{16}$ J. Rammer and H. Smith, Rev. Mod. Phys. 58, 323 (1986).

${ }^{17}$ At low temperatures the characteristic energy scale of the system is $|\Delta|$. Hence perturbation theory holds only when $\delta \mu \ll|\Delta|$. On the other hand, in the sliding regime $\delta \mu_{T} \ll \delta \mu$ which can only be satisfied if $\delta \mu_{T} \ll|\Delta|$. Therefore at low temperatures where $\delta \mu_{T}$ is not negligible, perturbation theory is only valid in the limit of weak pinning, i.e., $\delta \mu_{T} \ll|\Delta|$.

${ }^{18}$ B. Rejaei (unpublished).

${ }^{19}$ Note that in Andreev reflection exactly the opposite happens: charge is transferred from the normal metal to the superconductor while momentum is conserved. 